\title{
Efficacy and Safety of Anlotinib Monotherapy as Third-Line Therapy for Elderly Patients with Non-Small Cell Lung Cancer: A Real-World Exploratory Study
}

\author{
Hong-Tao Jiang \\ Wei Li \\ Biao Zhang \\ Qiang Gong \\ Hai-Ling Qie
}

Department of Thoracic Surgery, Affiliated Hospital of Hebei University, Baoding, 071000, People's Republic of China
Correspondence: Hai-Ling Qie; Qiang

Gong

Department of Thoracic Surgery,

Affiliated Hospital of Hebei University,

No. 212, Yu-Hua East Road, Lian-Chi

District, Baoding, 071000, People's

Republic of China

Tel +86 I5930757546; +86 I8903363263

Email qiehailing@163.com;

5751564@qq.com
Purpose: The present study was to investigate the real-world efficacy and safety of anlotinib monotherapy as third-line therapy for elderly patients with advanced non-small cell lung cancer (NSCLC).

Methods: A total of 83 elderly patients ( $>65$ years) with NSCLC who were progressed at least two lines of previous systemic therapy were recruited retrospectively. Patients were treated with anlotinib monotherapy (12 mg or $10 \mathrm{mg}$ ). Efficacy of anlotinib was assessed and all the patients were followed up regularly. Adverse reactions were collected during anlotinib administration. Univariate analysis was performed using Log rank test and multivariate analysis was adjusted by Cox regression analysis. Additionally, prognostic analysis according to common adverse reactions was implemented to identify the potential clinical significance.

Results: The best overall response of the 83 elderly patients during anlotinib monotherapy indicated that partial response (PR) was observed in six patients, stable disease (SD) was noted in 59 patients, and progressive disease (PD) was reported in 18 patients. Consequently, the objective response rate (ORR) was 7.2\% (95\% CI=2.7-15.1\%) and the disease control rate (DCR) was $78.3 \%(95 \% \mathrm{CI}=67.9-86.6 \%)$. The median progression-free survival (PFS) of the 83 elderly patients with NSCLC was 4.2 months $(95 \% \mathrm{CI}=3.51-4.89)$. Furthermore, the median overall survival (OS) of the 83 patients was 9.6 months $(95 \% \mathrm{CI}=6.65-12.55)$. The safety profile suggested that the relatively common adverse reactions of the elderly patients with ES-SCLC receiving anlotinib therapy were hypertension (49.4\%), fatigue (45.8\%), and hand-foot syndrome (39.8\%). Interestingly, association analysis exhibited that the median PFS of patients with hypertension and non-hypertension was 4.5 and 3.0 months, respectively $\left(\chi^{2}=6.306, P=0.012\right)$.

Conclusion: Anlotinib monotherapy demonstrated a satisfactory efficacy and tolerable safety profile in third-line settings for elderly patients with advanced NSCLC. Patients who experienced a hypertension adverse reaction induced by anlotinib therapy might confer superior PFS. The conclusion should be validated in prospective clinical trials subsequently. Keywords: elderly non-small cell lung cancer, anlotinib, efficacy, prognosis, safety, biomarker

\section{Introduction}

Lung cancer is the most common solid tumor all over the world, with approximately 815,000 new cases and 715,000 deaths in China annually. ${ }^{1}$ Non-small cell lung cancer (NSCLC) accounts for approximately $85 \%$ among lung cancer. ${ }^{2}$ 
Therefore, it is estimated that there are approximately 693,000 new cases and 608,000 deaths of NSCLC in China currently. To our knowledge, most patients were diagnosed with locally advanced or metastatic disease initially. ${ }^{3}$ Recent years had witnessed remarkable achievements regarding the immunotherapy and targeted drugs for EGFR, ALK, and ROS1, thus rendering NSCLC the most successful cancer in precision medicine. ${ }^{4}$ Angiogenesis was of utmost importance in the growth of tumor and was identified as an important therapeutic strategy for solid tumors according to the theory of professor Folkman since $1971 .^{5}$ Therefore, recent years had witnessed that antiangiogenic targeted drugs were developed and exhibited potential anticancer activity for advanced NSCLC. Bevacizumab and ramucirumab demonstrated sufficient survival benefit for patients with advanced NSCLC both in first-line and second-line treatment. ${ }^{6}$ As an antiangiogenic multi-targeted tyrosine kinase inhibitor (TKI), anlotinib had become a new standard of care as third-line therapy in China since 2018 according to a ALTER0303 Phase III clinical trial. ${ }^{7}$

To our knowledge, the prevalence of NSCLC decreased in younger patients and increased in elderly patients recently. ${ }^{8}$ A previous study indicated that approximately $50 \%$ patients with NSCLC were diagnosed among the population aged $\geq 65$ years, suggesting the necessity for appropriate therapeutic options in the elderly subgroup. ${ }^{9}$ Unfortunately, despite the increasing incidence and prevalence of tumors among the elderly population, patients older than 75 years accounted for less than $10 \%$ of subjects recruited in the national cancer institute cooperative group studies. Therefore, elderly patients with NSCLC were under-represented in clinical trials. ${ }^{10}$ Usually, the scantiness of elderly patients who participated in trial registration could be attributed to various factors: advanced age, relatively poor performance status, lack of adequate social support, cognitive dysfunction, and the presence of multiple comorbid conditions and the fact of unwillingness to accept increased toxicity. Consequently, in view of the strict age eligibility criteria in most clinical trials, comparatively scant medical evidence was available as subsequent-line treatment for elderly patients with NSCLC clinically. ${ }^{11}$ Regarding the studies of anlotinib in NSCLC, the age of the patients included in ALTER0303 ranged from 18-75 with patients $>70$ years accounting for only $8.4 \%{ }^{7}$ Another retrospective study regarding anlotinib in NSCLC who failed the standard therapy included 150 patients with the majority age $<65$ years. ${ }^{12}$ Collectively, clinical evidence regarding the efficacy and safety of anlotinib as third-line therapy for elderly patients with advanced NSCLC was absent and insufficient.

It should be noticed that the overall response of antiangiogenic targeted-drugs was disappointing. Objective response rate (ORR) of sorafenib, anlotinib, and apatinib monotherapy as third-line therapy for advanced NSCLC was only $4.9 \%, 9.2 \%$, and $4.0 \%$, respectively. ${ }^{13}$ Therefore, great individual difference regarding the efficacy of antiangiogenic targeted drugs existed clinically. ${ }^{14}$ As a result, exploration of biomarkers that could identify the efficacy of anlotinib was of valuable clinical implications currently.

Consequently, the present study was to investigate the efficacy and safety of anlotinib in third-line therapy for elderly patients with advanced NSCLC and the clinical significance according to common adverse reactions.

\section{Patients and Methods Study Design and Eligibility Criteria}

The present study was designed as a retrospective analysis. Patients with treatment refractory advanced NSCLC from June 2018 to March 2021 in the Department of Thoracic Surgery of Affiliated Hospital of Hebei University were participated in this study consecutively. Inclusion criteria included: 1) histology confirmed diagnosis of NSCLC; 2) pathological staging of IIIb or IV; 3 ) age $\geq 65$ years (elderly classification of World Health Organization); 4) the Eastern Cooperative Oncology Group (ECOG) performance status (PS) of 0-2 score; 5) anlotinib monotherapy was administered for patients with NSCLC who failed at least two lines of systemic therapy previously; and 6) at least one measurable target lesion according to response evaluation criteria in solid tumors (RECIST 1.1) to exhibit the drug response. Exclusion criteria manifested as: 1) active stage or uncontrolled central nervous system metastases; 2) concomitant with another cancer or serious disease that could compromise the survival of the patients in the opinion of the investigators; 3) previous exposure to anlotinib therapy, however, previous other antiangiogenic targeted drugs exposure was permitted; 4) presence of symptom for hemoptysis $>50 \mathrm{~mL} /$ day; and 5) data of efficacy evaluation was not available. However, patients who were lost to follow-up were included and suitable for survival analysis. A flow chart of this retrospective study is illustrated in Figure 1. Finally, a total of 83 elderly patients with advanced NSCLC were enrolled in this study. The primary endpoint of the present study was progression-free survival (PFS), the secondary endpoints were ORR, disease control rate (DCR), 
overall survival (OS), safety profile, and association analysis between PFS and adverse reaction status. The protocol of the present study was approved by the Ethics Committee of the Affiliated Hospital of Hebei University, which was conducted in accordance with the Declaration of Helsinki and Good Clinical Practice guidelines. Informed consent was signed from each enrolled patient and medical data of the subjects were confidential at the highest level.

\section{Therapeutic Regimens}

Patients were treated with anlotinib (administered orally once daily for day $1-14$, stopped for 7 days and every 21 days as one cycle) at an initial dosage of $12 \mathrm{mg}$ or $10 \mathrm{mg}$ before breakfast with warm water. Dosages of $12 \mathrm{mg}$ or $10 \mathrm{mg}$ depended on the investigators' judgment according to the physical conditions of the elderly patients. The majority of the patients were treated with anlotinib of $12 \mathrm{mg}$. Additionally, dosage adjustment to $10 \mathrm{mg}$ or $8 \mathrm{mg}$ was permitted according to the adverse reactions during the treatment. Specifically, when grade 3 or 4 adverse reactions occurred during anlotinib administration, the treatment of anlotinib should be suspended until the adverse reaction recovered to $<$ grade 2 . Then the initial dosage of anlotinib might be continued if the patient could tolerate it or dosage adjustment was adopted (from $12 \mathrm{mg}$ to $10 \mathrm{mg}$ or from $10 \mathrm{mg}$ to $8 \mathrm{mg}$ ) according to the actual situation or the judgment of the investigator. The treatment could be terminated when any life-threatening toxic reaction occurred. Patients continued the treatment until disease progression or intolerable adverse reactions.

A total of 260 patients with advanced NSCLC underwent eligibility screening retrospectively from June 2018 to March 2021

156 patients failed to meet the eligibility criteria

$>97$ patients were out of age criterion ( $<60$ years)

38 patients failed to receive anlotinib treatment

13 patients were out of ECOG criterion

8 patients were not available for the measurable target lesion

A total of 104 patients with NSCLC met the eligibility criteria

21 patients met the exclusion criteria

6 patients were in active stage or untreated central nervous system metastases

$>8$ patients were concomitant with other tumors or serious diseases

> 5 patients were absence of the data for efficacy evaluation information

$>2$ patients were presence of the symptom for hemoptysis

A total of 83 elderly patients with advanced NSCLC were recruited in the retrospective study

Figure I Flow chart of the retrospective study of anlotinib monotherapy as third-line therapy for elderly patients with non-small cell lung cancer. 


\section{Response Assessment and Adverse Reaction}

Drug response was evaluated according to RECIST version 1.1 criteria by investigator assessment using chest computed tomography (CT) scans or nuclear magnetic resonance imaging (MRI) at baseline and during anlotinib treatment. Change of target lesions was assessed every two cycles or necessary in the opinion of investigators. The results of each imaging assessment were recorded and analyzed to document the best overall response of the patients. ${ }^{15}$ Subsequently, follow-up was implemented using phone monthly, and the death status of the patients was mainly obtained through the communication with the relatives of the patients. The last follow-up date of the present study was July, 2021.

Regarding the safety profile, adverse reactions during treatment were evaluated using Common Terminology Criteria for Adverse Events (CTCAE) version 4.03 criteria to document adverse reactions that might be drugrelated. ${ }^{16}$ Clinical significance according to common adverse reactions was performed and analyzed. Hypertension was the most common adverse reaction, which was found to be of positive relevance to the prognosis during the preliminary analysis. Therefore, subsequent correlation analysis was mainly implemented between hypertension status and PFS.

\section{Statistical Analysis}

Efficacy data manifested as ORR and DCR. ORR was defined as the proportion of patients with complete response (CR) plus partial response (PR) in total patients. DCR was defined as the proportion of patients with $\mathrm{CR}$ plus PR and stable disease (SD) in total patients. All the variables were statistically analyzed using SPSS version 25.0 (IBM, USA). Prognostic data presented as PFS and OS. PFS was defined as the duration from the onset of anlotinib administration to disease progression or death, whichever occurred first. OS was defined as the duration from the onset of anlotinib administration to death of the patients from any cause. For those without progression or death events at the end of the study, survival end points were censored at the date of last follow-up.

Statistical difference of proportion variables and continuous variables according to hypertension status was analyzed using the Chi-square test and the Mann-Whitney $\mathrm{U}$ non-parametric test, respectively. Kaplan-Meier curves were adopted using Stata version 14.0 to compare the differences in PFS according to common adverse reactions status. The survival difference was calculated using Log rank test. Cox multivariate analysis with the stepwise method was adopted to identify the prognostic influencers for PFS to produce the hazard ratio (HR) and $95 \%$ confidence interval (CI). Statistical significance was accepted when $P<0.05$.

\section{Results}

\section{Baseline Characteristics of the 83 Elderly Patients with Advanced NSCLC According to Hypertension Status}

Baseline characteristics of the 83 elderly patients with advanced NSCLC are exhibited in Table 1. Subjects included were elderly patients with the median age of 76 years (range $=65-87$ years). A total of 59 patients were male and 24 patients were female. Pathological stage IV was observed in 76 patients $(91.6 \%)$. ECOG performance status $0-1$ score was noted in 39 patients. Non-smokers/former smokers were reported in 65 patients $(78.3 \%)$. The most common histology of the patients was adenocarcinoma, with 62 cases (74.7\%), and squamous cell carcinoma, with 21 cases $(25.3 \%)$. Numbers of metastases sites of $\leq 3$ and $>3$ were seen in 52 and 31 cases, respectively. Driver gene positive mutation was observed in 31 patients $(37.3 \%)$. Interestingly, previous treatment with second line and subsequent line was noted in 21 patients and 62 patients, respectively. Besides, a total of 44 patients $(53.0 \%)$ had received targeted drug therapy previously, including EGFRTKI and bevacizumab-based regimens. It should be noticed that eight patients $(9.6 \%)$ were administered with antiangiogenic TKI treatment previously. Furthermore, a total of 19 patients had treatment with immunotherapy previously. Anlotinib was administered with an initial dosage of $12 \mathrm{mg}$ or $10 \mathrm{mg}$ in 69 and 14 patients, respectively.

Additionally, patients with hypertension were observed in 41 cases $(49.4 \%)$. As presented in Table 1, baseline characteristics of patients according to hypertension status were balanced and comparable $(P>0.05)$.

\section{Efficacy of the 83 Elderly Patients with Advanced NSCLC Receiving Anlotinib Monotherapy}

All the 83 elderly patients with advanced NSCLC were able to evaluate the efficacy. The best overall response of each enrolled patient during anlotinib administration 
Table I Baseline Characteristics of the 83 Elderly Patients with Advanced NSCLC According to Hypertension Status

\begin{tabular}{|c|c|c|c|c|c|}
\hline \multirow[t]{2}{*}{ Characteristics } & \multirow{2}{*}{$\begin{array}{c}\text { Total Patients }(\mathrm{N}=83 \text {, } \\
\%)\end{array}$} & \multicolumn{2}{|c|}{ Hypertension Status } & \multirow[t]{2}{*}{$\chi^{2}$} & \multirow[t]{2}{*}{$P$} \\
\hline & & $\begin{array}{l}\text { Hypertension } \\
\qquad(\mathrm{N}=4 \mathrm{I})\end{array}$ & $\begin{array}{l}\text { Non-Hypertension } \\
\qquad(\mathbf{N}=42)\end{array}$ & & \\
\hline \multicolumn{6}{|l|}{ Age (years) } \\
\hline Median (range) & $76(65-87)$ & $75(65-85)$ & $76(65-87)$ & NA & 0.615 \\
\hline \multicolumn{6}{|l|}{ Gender } \\
\hline Male & $59(71.1)$ & $29(70.7)$ & $30(71.4)$ & 0.005 & 0.944 \\
\hline Female & $24(28.9)$ & $12(29.3)$ & $12(28.6)$ & & \\
\hline \multicolumn{6}{|l|}{ Pathological stage } \\
\hline IIlb & $7(8.4)$ & $3(7.3)$ & $4(9.5)$ & 0.131 & 0.718 \\
\hline IV & $76(91.6)$ & $38(92.7)$ & $38(90.5)$ & & \\
\hline \multicolumn{6}{|l|}{ ECOG PS score } \\
\hline $0-1$ & $39(47.0)$ & $20(48.8)$ & $19(45.2)$ & 0.105 & 0.746 \\
\hline 2 & $44(53.0)$ & $21(5 \mid .2)$ & $23(54.8)$ & & \\
\hline \multicolumn{6}{|l|}{ Smoking status } \\
\hline Nonsmoker/former smoker & $65(78.3)$ & $31(75.6)$ & $34(80.9)$ & 0.349 & 0.555 \\
\hline Smoker & $18(21.7)$ & $10(24.4)$ & $8(19.1)$ & & \\
\hline \multicolumn{6}{|l|}{ Histology } \\
\hline Adenocarcinoma & $62(74.7)$ & $31(75.6)$ & 31 (73.8) & 0.036 & 0.850 \\
\hline Squamous cell carcinoma & $21(25.3)$ & $10(24.4)$ & II (26.2) & & \\
\hline \multicolumn{6}{|l|}{ Number of metastases sites } \\
\hline$\leq 3$ & $52(62.7)$ & $26(63.4)$ & $26(61.9)$ & 0.020 & 0.887 \\
\hline$>3$ & $31(37.3)$ & $15(36.6)$ & $16(38.1)$ & & \\
\hline \multicolumn{6}{|l|}{ Driver gene mutation status ${ }^{a}$} \\
\hline Positive & $31(37.3)$ & $15(36.6)$ & $16(38.1)$ & 0.020 & 0.887 \\
\hline Negative & $52(62.7)$ & $26(63.4)$ & $26(61.9)$ & & \\
\hline \multicolumn{6}{|l|}{ Lines of previous treatment } \\
\hline Second line & $21(25.3)$ & $9(22.0)$ & $12(28.6)$ & 0.481 & 0.488 \\
\hline Subsequent line & $62(74.7)$ & $32(78.0)$ & $30(71.4)$ & & \\
\hline \multicolumn{6}{|c|}{ History of targeted drug therapy } \\
\hline Yes & $44(53.0)$ & $21(5 \mid .2)$ & $23(54.8)$ & 0.105 & 0.746 \\
\hline No & $39(47.0)$ & $20(48.8)$ & $19(45.2)$ & & \\
\hline \multicolumn{6}{|c|}{$\begin{array}{l}\text { History of antiangiogenic TKI } \\
\text { therapy }\end{array}$} \\
\hline Yes & $8(9.6)$ & $4(9.8)$ & $4(9.5)$ & 0.001 & 0.971 \\
\hline No & $75(90.4)$ & $37(90.2)$ & $38(90.5)$ & & \\
\hline \multicolumn{6}{|l|}{ History of immunotherapy } \\
\hline Yes & $19(22.9)$ & $9(22.0)$ & $10(23.8)$ & 0.041 & 0.840 \\
\hline No & $64(77.1)$ & $32(78.0)$ & $32(76.2)$ & & \\
\hline \multicolumn{6}{|c|}{ Initial dosage of anlotinib (mg) } \\
\hline 12 & $69(83.1)$ & 34 (82.9) & 35 (83.3) & 0.002 & 0.961 \\
\hline 10 & $14(16.9)$ & $7(17.1)$ & $7(16.7)$ & & \\
\hline
\end{tabular}

Note: a Driver gene mutation status was EGFR mutation or ALK or ROSI rearrangement.

Abbreviations: NSCLC, non-small cell lung cancer; NA; not available; ECOG, Eastern Cooperative Oncology Group; PS, performance status; TKI, tyrosine kinase inhibitor. 


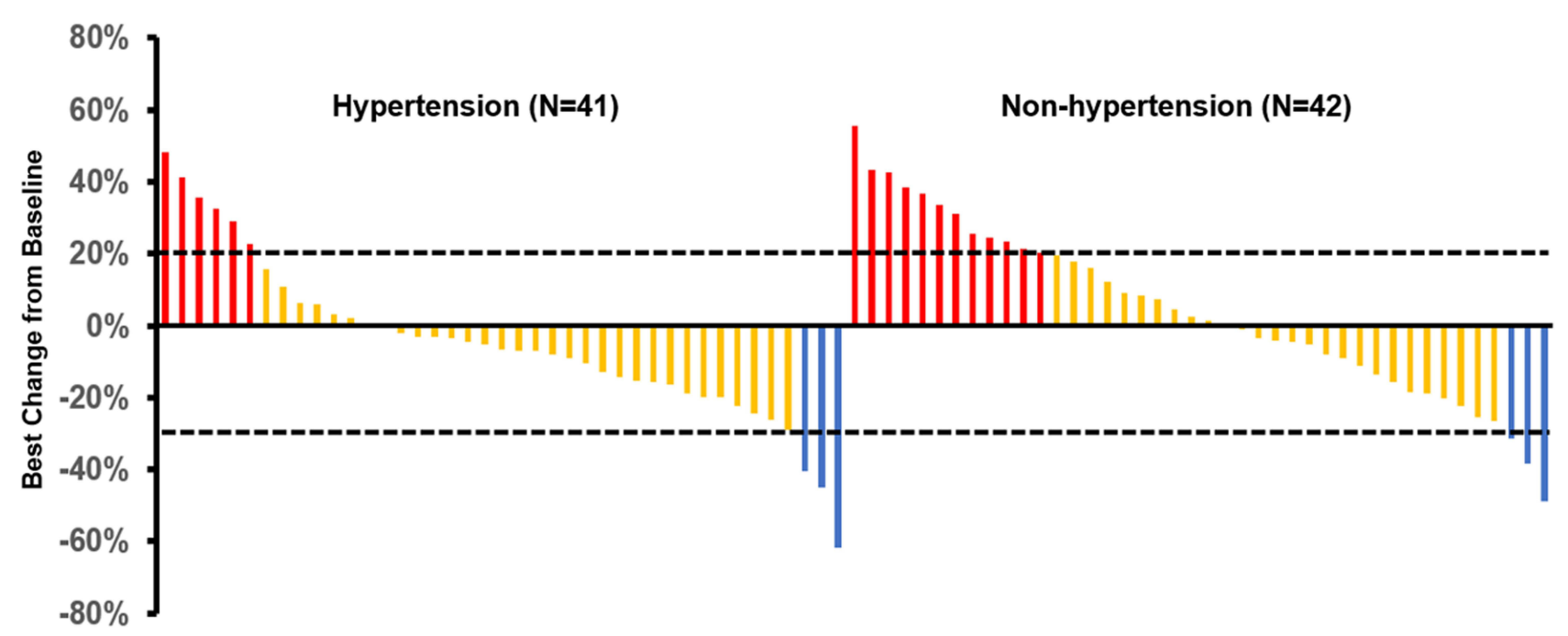

Figure 2 Waterfall plot for the best percentage change in target lesion size of the 83 elderly patients with non-small cell lung cancer receiving anlotinib monotherapy according to hypertension status.

indicated that $\mathrm{CR}$ was seen in no patients, $\mathrm{PR}$ was observed in six patients, SD was noted in 59 patients and progressive disease (PD) was reported in 18 patients according to RECIST version 1.1. Consequently, the ORR of the 83 elderly patients was $7.2 \%(95 \% \mathrm{CI}=2.7-$ $15.1 \%)$, DCR was $78.3 \% \quad(95 \% \quad \mathrm{CI}=67.9-86.6 \%)$. Waterfall plot for the best percentage change in target lesion of the 83 elderly patients according to hypertension status is illustrated in Figure 2. Accordingly, ORR of patients with hypertension and non-hypertension was $7.3 \%$ and $7.1 \%$, respectively $(P=0.976)$. And DCR of patients with hypertension and non-hypertension was $85.5 \%$ and $71.4 \%$, respectively $(P=0.123)$. Additionally, CT scan for the change of target lesions in one elderly patient with advanced NSCLC before and after anlotinib administration was illustrated in Figure 3. Administration with anlotinib was effective prominently and the target lesion declined significantly.

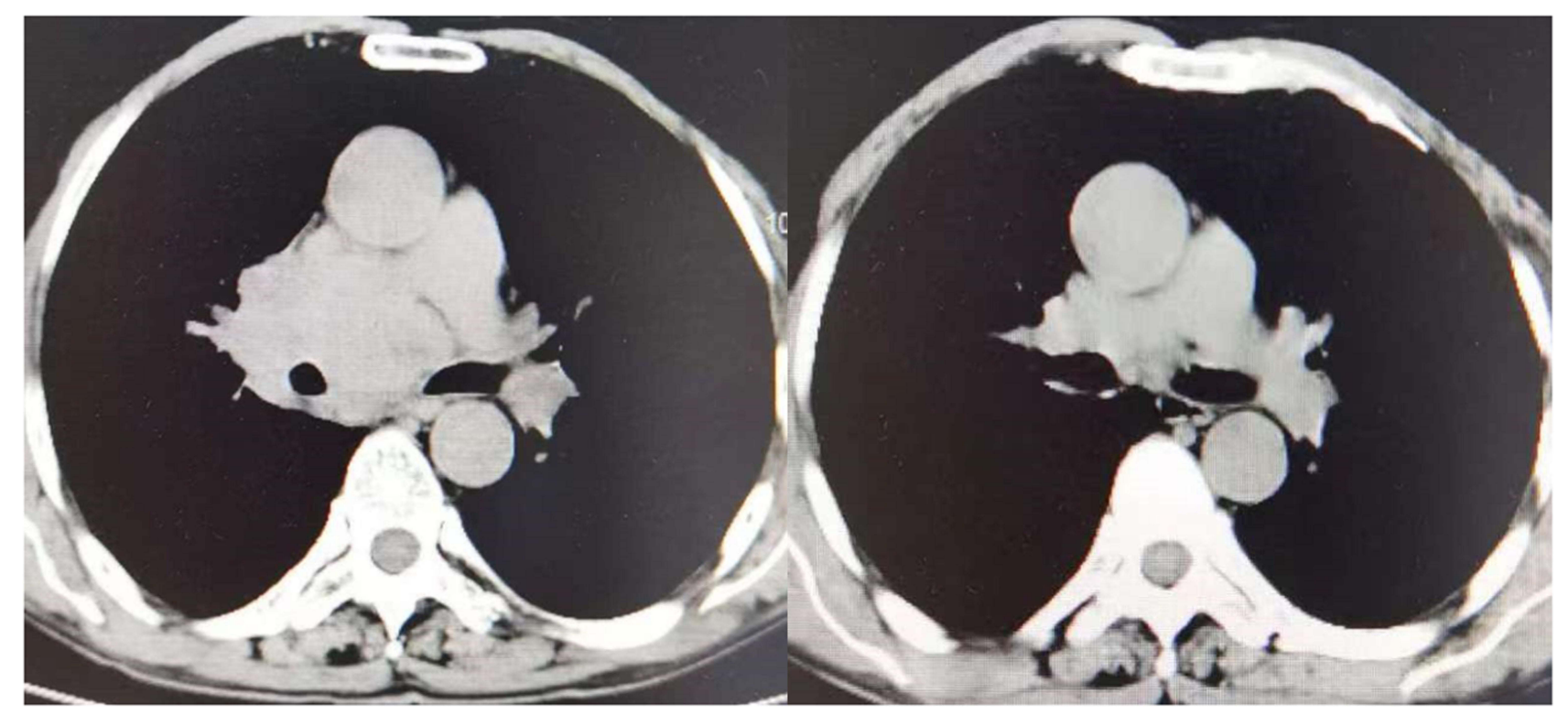

Figure 3 The CT scan results for the changes of target lesions in one elderly patient with non-small cell lung cancer before and after the treatment of anlotinib [follow-up date: December 5, 2018 (left) and March 4, 2019 (right)]. 

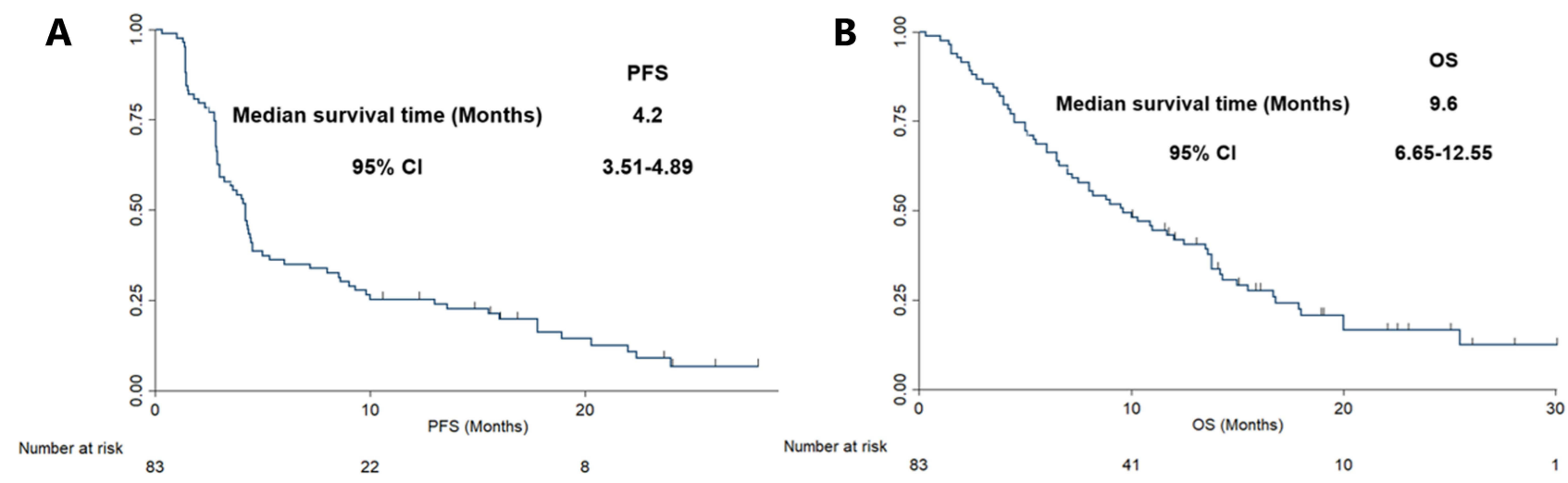

Figure 4 Progression-fee survival (A) and Overall Survival (B) of the 83 elderly patients with treatment refractory non-small cell lung cancer receiving anlotinib monotherapy.

\section{Prognosis of the 83 Elderly Patients with Advanced NSCLC Receiving Anlotinib Monotherapy}

Regarding the prognostic data, the median follow-up duration for all the patients from the date of enrollment to the last follow-up date was 8.6 months (follow-up range $=0.3-30.0$ months). As exhibited in Figure 4A, the median PFS of the 83 elderly patients with advanced NSCLC receiving anlotinib monotherapy was 4.2 months (95\% $\mathrm{CI}=3.51-4.89)$. Furthermore, prognostic significance of various baseline characteristics was also analyzed with the primary endpoint-PFS. Univariate analysis investigated the median PFS and 95\% CI according to different baseline characteristic subgroups. Interestingly, as exhibited in Table 2, ECOG PS score and number of metastases sites were strikingly associated with PFS in univariate analysis. The median PFS of patients with PS 0-1 score and 2 score was 5.0 months and 3.1 months, respectively $(P=0.009)$. Besides, patients with a number of metastases sites $\leq 3$ conferred a longer PFS than patients with a number of metastases sites $>3$ (median PFS: 4.8 versus 3.6 months, $P=0.021$ ). The other baseline characteristics showed balanced efficacy regarding PFS of anlotinib monotherapy and no significant statistical difference was observed $(P>0.05)$.

Furthermore, as shown in Figure 4B, the comparatively long follow-up duration yielded the mature survival data, which indicated that the median OS of the 83-patients cohort was 9.6 months $(95 \% \mathrm{CI}=6.65-12.55)$.

\section{Safety Profile of the 83 Elderly Patients with Advanced NSCLC Receiving Anlotinib Monotherapy}

The maximum toxicity of the 83 elderly patients experienced during anlotinib monotherapy were collected and analyzed in the present study. As shown in Table 3, the common drug-related adverse reactions were hypertension (49.4\%), fatigue (45.8\%), hand-foot syndrome (HFS, $39.8 \%$ ), diarrhea $(33.7 \%)$, loss of weight $(31.3 \%)$, decreased appetite $(21.7 \%)$, hematological toxicity (18.1\%), hypertriglyceridemia (13.3\%), AST/ALT elevation $(9.6 \%)$, and hemoptysis $(6.0 \%)$. Furthermore, the grade $\geq 3$ adverse reactions were observed in hypertension (18.1\%), fatigue $(6.0 \%)$, HFS (7.2\%), diarrhea (3.6\%), loss of weight $(4.8 \%)$, decreased appetite $(1.2 \%)$, hematological toxicity $(2.4 \%)$, hypertriglyceridemia $(1.2 \%)$, AST/ ALT elevation (1.2\%), and hemoptysis (1.2\%). Total adverse reactions were tolerable and controllable.

\section{Clinical Implication According to Hypertension Status}

As exhibited in Table 3, a total of 41 patients were observed to experience hypertension adverse reaction during anlotinb treatment. Accordingly, the PFS of the 83 elderly patients with advanced NSCLC according to hypertension status is illustrated in Figure 5. The median PFS of patients with hypertension and non-hypertension was 4.5 months $(95 \% \mathrm{CI}=0.86-8.14)$ and 3 months $(95 \%$ $\mathrm{CI}=1.71-4.29)$, respectively. The difference was statistically significant $\left(\chi^{2}=6.306, P=0.012\right)$.

Furthermore, Cox multivariate analysis was performed to identify the independent significance of hypertension status. Those factors (ECOG PS score, number of metastases sites and hypertension status) showed positive relevance to PFS in univariate analysis $(P<0.05)$ was included in the multivariate analysis accordingly. The results of multivariate analysis are illustrated in Table 4, and a significant statistical difference was still observed, which indicated that hypertension status 
Table 2 Univariate Analysis for PFS of the 83 Elderly Patients with Advanced NSCLC According to Baseline Characteristics

\begin{tabular}{|c|c|c|c|c|}
\hline Characteristics & $\mathbf{N}$ & $\begin{array}{l}\text { Median PFS } \\
\text { (Months) }\end{array}$ & $95 \% \mathrm{Cl}$ & $P$ \\
\hline \multicolumn{5}{|l|}{ Age (Years) } \\
\hline$<76$ & 38 & 4.5 & $3.64-5.36$ & 0.435 \\
\hline$\geq 76$ & 45 & 4.0 & $3.32-4.68$ & \\
\hline \multicolumn{5}{|l|}{ Gender } \\
\hline Male & 59 & 3.9 & $3.21-4.59$ & 0.318 \\
\hline Female & 24 & 4.6 & $3.79-5.41$ & \\
\hline \multicolumn{5}{|l|}{ Pathological stage } \\
\hline IIIb & 7 & 4.8 & $4.01-5.59$ & 0.613 \\
\hline IV & 76 & 4.2 & $3.49-4.91$ & \\
\hline \multicolumn{5}{|l|}{ ECOG PS score } \\
\hline $0-1$ & 39 & 5.0 & $1.13-8.87$ & 0.009 \\
\hline 2 & 44 & 3.1 & $|.79-4.4|$ & \\
\hline \multicolumn{5}{|l|}{ Smoking status } \\
\hline Nonsmoker/former smoker & 65 & 4.2 & $3.37-5.03$ & 0.734 \\
\hline Smoker & 18 & 4.2 & $3.4 I-4.99$ & \\
\hline \multicolumn{5}{|l|}{ Histology } \\
\hline Adenocarcinoma & 62 & 4.4 & $3.73-5.07$ & 0.411 \\
\hline Squamous cell carcinoma & 21 & 4.0 & $3.19-4.81$ & \\
\hline \multicolumn{5}{|l|}{ Number of metastases sites } \\
\hline$\leq 3$ & 52 & 4.8 & $2.25-7.35$ & 0.021 \\
\hline$>3$ & 31 & 3.6 & $2.09-5.11$ & \\
\hline \multicolumn{5}{|l|}{ Driver gene mutation status } \\
\hline Positive & 31 & 4.4 & $3.62-5.18$ & 0.616 \\
\hline Negative & 52 & 4.2 & $3.54-4.86$ & \\
\hline \multicolumn{5}{|l|}{ Lines of previous treatment } \\
\hline Second line & 21 & 4.2 & $3.58-4.82$ & 0.519 \\
\hline Subsequent line & 62 & 4.0 & $3.31-4.69$ & \\
\hline \multicolumn{5}{|l|}{$\begin{array}{l}\text { History of targeted drug } \\
\text { therapy }\end{array}$} \\
\hline Yes & 44 & 4.4 & $3.59-5.21$ & 0.439 \\
\hline No & 39 & 3.9 & $3.08-4.72$ & \\
\hline \multicolumn{5}{|l|}{ History of antiangiogenic } \\
\hline \multicolumn{5}{|l|}{ TKI therapy } \\
\hline Yes & 8 & 3.9 & $3.27-4.53$ & 0.562 \\
\hline No & 75 & 4.2 & $3.41-4.99$ & \\
\hline \multicolumn{5}{|l|}{ History of immunotherapy } \\
\hline Yes & 19 & 4.8 & $4.04-5.56$ & 0.313 \\
\hline No & 64 & 4.0 & $3.18-4.82$ & \\
\hline \multicolumn{5}{|l|}{$\begin{array}{l}\text { Initial dosage of anlotinib } \\
\text { (mg) }\end{array}$} \\
\hline 12 & 69 & 4.5 & $3.77-5.23$ & 0.432 \\
\hline 10 & 14 & 4.0 & $3.21-4.79$ & \\
\hline
\end{tabular}

Abbreviations: NSCLC, non-small cell lung cancer; NA; not available; ECOG, Eastern Cooperative Oncology Group; PS, performance status; TKI, tyrosine kinase inhibitor; $\mathrm{Cl}$, confidence interval. 
Table 3 Safety Profile of the 83 Elderly Patients with Advanced NSCLC Receiving Anlotinib Monotherapy

\begin{tabular}{|c|c|c|c|}
\hline $\begin{array}{l}\text { Adverse } \\
\text { Reactions }\end{array}$ & $\begin{array}{l}\text { Total } \\
(\mathrm{N}, \%)\end{array}$ & $\begin{array}{c}\text { Grade I-2 } \\
(\mathrm{N}, \%)\end{array}$ & $\begin{array}{c}\text { Grade } \geq 3 \\
(\mathbf{N}, \%)\end{array}$ \\
\hline Hypertension & $41(49.4)$ & $26(31.3)$ & $15(18.1)$ \\
\hline Fatigue & $38(45.8)$ & $33(39.8)$ & $5(6.0)$ \\
\hline Hand-foot syndrome & $33(39.8)$ & $27(32.5)$ & $6(7.2)$ \\
\hline Diarrhea & $28(33.7)$ & $25(30.1)$ & $3(3.6)$ \\
\hline Loss of weight & $26(31.3)$ & $22(26.5)$ & $4(4.8)$ \\
\hline Decreased appetite & $18(21.7)$ & $17(20.5)$ & $\mathrm{I}(\mathrm{I} .2)$ \\
\hline $\begin{array}{l}\text { Hematological } \\
\text { toxicity }\end{array}$ & $15(18.1)$ & $13(15.7)$ & $2(2.4)$ \\
\hline Hypertriglyceridemia & II (I3.3) & $10(12.1)$ & $\mathrm{I}(\mathrm{I} .2)$ \\
\hline AST/ALT elevation & $8(9.6)$ & $7(8.4)$ & $\mathrm{I}(\mathrm{I} .2)$ \\
\hline Hemoptysis & $5(6.0)$ & $4(4.8)$ & $\mathrm{I}(\mathrm{I} .2)$ \\
\hline
\end{tabular}

Abbreviations: NSCLC, non-small cell lung cancer; AST, aspartate amino transferase; ALT, alanine aminotransferase.

was an independent factor for PFS $(\mathrm{HR}=0.69, P=0.021)$. Additionally, ECOG score was also noted to be an independent factor for PFS $(\mathrm{HR}=0.63, P=0.015)$. Besides, the number of metastases was also an independent factor for PFS $(\mathrm{HR}=0.75, P=0.035)$.

\section{Discussion}

The present study was the first retrospective study that highlighted real-world evidence regarding the efficacy and safety of anlotinib monotherapy as third-line therapy for elderly patients with NSCLC. Simultaneously, clinical implications according to hypertension status suggested that hypertension induced by anlotinib monotherapy might be associated with superior PFS. Collectively, anlotinib could be effective and safe for elderly patients with treatment refractory NSCLC.

To our knowledge, recent years have witnessed the increase of elderly patients with NSCLC in China dramatically. ${ }^{17}$ It was reported that the decreases in lean body mass, hepatic blood flow, and renal function was involved in the distribution, metabolism, and excretion of the targeted drugs to some extent. ${ }^{18}$ Therefore, elderly patients were always selected with caution and strict restrictions on age criteria had rejected the elderly patients largely in numerous clinical trials, ${ }^{19}$ which resulted in a great discrepancy between the proportion of elderly patients in the total population and the proportion in clinical trials regarding lung cancer: $67 \%$ versus $35 \%{ }^{20}$ Therefore, under-representation was especially significant for patients $\geq 75$ years. $^{21}$ Consequently, elderly patients with advanced NSCLC were urgently needed for effective and safe drugs both in clinical trials and clinical practice.

Despite the fact that recent years had witnessed that rapid progress of targeted therapy, immunotherapy and antiangiogenic targeted drugs strikingly prolonged the survival of

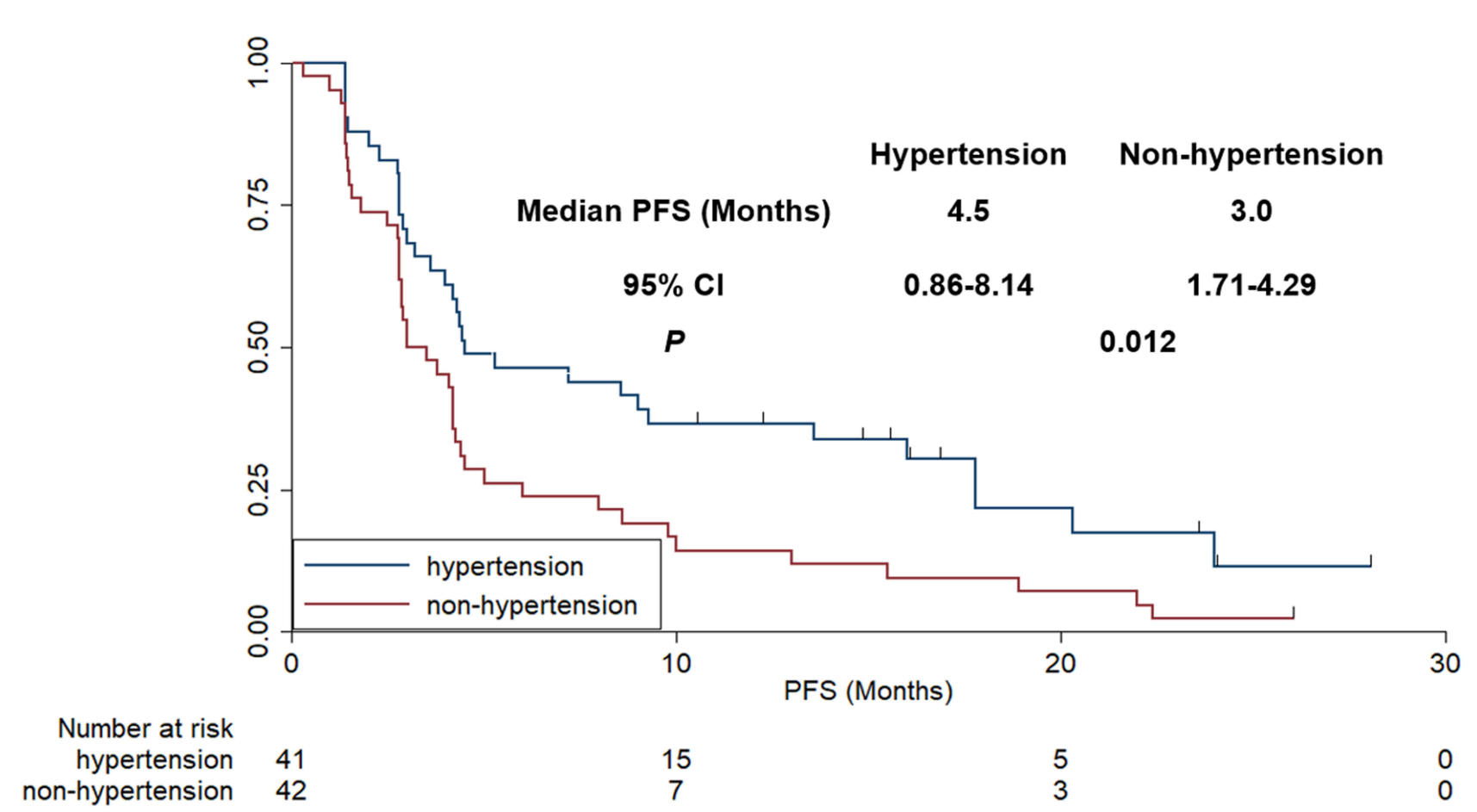

Figure 5 Progression-free survival of the 83 elderly patients with treatment refractory non-small cell lung cancer according to hypertension status. 
Table 4 Multivariate Cox Analysis for PFS According to Baseline Characteristics and Hypertension Status

\begin{tabular}{|l|l|l|c|}
\hline Characteristics & HR & $95 \%$ CI & $P$ \\
\hline $\begin{array}{l}\text { ECOG PS score } \\
0-1 \text { vs 2 }\end{array}$ & 0.63 & $0.29-0.89$ & 0.015 \\
\hline $\begin{array}{l}\text { Number of metastases sites } \\
\leq 3 \text { vs >3 }\end{array}$ & 0.75 & $0.45-0.94$ & 0.035 \\
\hline $\begin{array}{l}\text { Hypertension status } \\
\text { Hypertension vs non-hypertension }\end{array}$ & 0.69 & $0.42-0.91$ & 0.021 \\
\hline
\end{tabular}

Abbreviations: ECOG, Eastern Cooperative Oncology Group; PS, performance status; $\mathrm{HR}$, hazard ratio; $\mathrm{Cl}$, confidence interval.

patients with NSCLC both in first-line and second-line therapy, most patients still progressed or failed the treatment eventually with relatively appropriate performance status to receive the subsequent therapy. ${ }^{22}$ However, treatment as a third-line or subsequent-line for patients with advanced NSCLC remained dismal. ${ }^{23}$ Encouragingly, anlotinib suppressed both tumor angiogenesis via inhibition of VEGFR, PDGFR, and FGFR and tumor proliferation through the target of c-Kit, Ret, and c-FMS. ${ }^{24}$ Consequently, anlotinib was approved for the treatment of NSCLC, ${ }^{7}$ SCLC, ${ }^{25}$ and soft tissue sarcoma in China since June 2018. ${ }^{26}$ Regarding the elderly patients with lung cancer who received anlotinib therapy, a previous study initiated by PF Song and colleagues included a total of 79 elderly patients with extensivestage small cell lung cancer aged 60 years old or above who were treated with $12 \mathrm{mg}$ anlotinib monotherapy. ${ }^{15}$ The results suggested that the preliminary efficacy and safety of anlotinib for elderly patients with previously treated ESSCLC was satisfactory, which exhibited that patients with lung cancer could stand a good chance to benefit from anlotinib monotherapy.

Overall response of anlotinib in elderly patients with advanced NSCLC exhibited that ORR and DCR were $7.2 \%$ and $78.3 \%$, respectively. Additionally, prognostic data indicated that the median PFS was 4.2 months $(95 \%$ $\mathrm{CI}=3.51-4.89$ ). Efficacy regarding ORR and DCR in our study was consistent with that in the ALTER0303 clinical trial. However, PFS in our study seemed to be slightly worse than that of the phase III clinical trial of anlotinib for advanced NSCLC initiated by BH Han and colleagues according to the ALTER0303 trial (ORR $=9.2 \%$, $\mathrm{DCR}=81.0 \%$ median PFS $=5.4$ months of anlotinib arm $){ }^{7}$ We speculated that the dominant reason could be attributed to the discrepancy of age between the two studies. The median ages in ALTER0303 and our study were 59 years and 76 years, respectively. After all, our study was designed specifically in elderly patients with a comparatively worse prognosis. ${ }^{27}$ The second reason could be the retrospective design of our study. To our knowledge, management of the patients and adherence of the therapy in a retrospective study was not sufficient and normative compared with the well-designed phase III clinical trial, which was proved in another retrospective study. ${ }^{28} \mathrm{~A}$ third reason could be attributed to the fact that patients with ECOG 2 score in our study accounted for a much higher proportion than those in the ALTER0303 trial $(53.0 \%$ versus $0.7 \%)$. To our knowledge, ECOG performance status was confirmed to be an independent prognostic factor to predict the survival among patients with NSCLC. ${ }^{29}$ Furthermore, Cox regression analysis in our study also suggested that patients with ECOG performance status 2 score were correlated with worse PFS independently. Another two retrospective studies regarding anlotinib monotherapy for patients with previously treated NSCLC were reported recently. They included a total of 52 and 206 patients both aged 18 years older. And the ORR, DCR, and median PFS were 10-16\%, 78-80\%, and 4.0-4.5 months, respectively. ${ }^{30,31}$ The subgroup analysis in their study suggested that the median PFS of patients $\geq 60$ years was 4.5 and 4.0 months and no statistical significance regarding PFS analysis was observed according to the age subgroup, which was consistent with that in our study and demonstrated that elderly patients could benefit from anlotinib monotherapy similar to the younger patients. Additionally, another independent factor that showed positive relevance to PFS identified in our study was the number of metastases sites. The results were also in concert with that in another retrospective study initiated by Cheng et al. ${ }^{32}$ Furthermore, the median OS of the 83 elderly patients with advanced NSCLC receiving anlotinib monotherapy was 9.6 months, which was close to that reported in the phase III clinical trial (ALTER0303, median OS=9.63 months of anlotinib arm) in spite of the fact that patients in our study were much older. The explanation could be attributed to the tendency that considerable immunotherapy drugs (PD-1 or PD-L1 inhibitors) were licensed in China since the approval of anlotinib in June 2018, which could contribute to the survival benefit for the elderly patients as subsequent treatment consecutively. Pembrolizumab, nivolumab, sintilimab, and camrelizumab were proved to be effective for previously treated patients with advanced NSCLC in subsequent-line therapy. ${ }^{33}$ Consequently, elderly patients with previously 
treated NSCLC were able to choose the immunotherapy as the subsequent-line treatment when they failed anlotinib administration, which might prolong the survival of the patients to some extent.

Regarding the safety profile of anlotinib in elderly patients, the most common adverse reactions during anlotinib administration manifested as hypertension, fatigue, HFS, diarrhea, and loss of weight, which were the frequently experienced adverse reactions of antiangiogenic targeted drugs clinically. ${ }^{33}$ However, it should be noted that the incidence of hypertension in our study seemed to be higher than that in the ALTER0303 trials, though a considerable proportion of the patients in our study were treated with the initial dosage of $10 \mathrm{mg}$ anlotinib, especially the incidence of grade $\geq 3$ hypertension $(18.1 \%$ versus $13.2 \%$ ). The possible explanation could be the elderly patients in our study. To our knowledge, elderly patients were often concomitant with medical and physiological characteristics that were susceptible to hypertension and other predisposition factors that might contribute to hypertension according to the previous research. ${ }^{34}$ That could explain the reason why the incidence of hypertension in elderly patients was higher when receiving anlotinib treatment. Interestingly, the relevance of hypertension status to PFS in our study identified the potential biomarker for anlotinib monotherapy among patients with advanced NSCLC clinically, which suggested that hypertension induced by anlotnib monotherapy could be used as a valuable biomarker to predict PFS of the patients. And the results were in concert with that of the previous retrospective study regarding another antiangiogenic TKIapatinib for patients with NSCLC to some extent. ${ }^{35,36}$ To our knowledge, hypertension might be one of the most common and most representative adverse reactions induced by the treatment of antiangiogenic targeted drugs that effected the VEGF/VEGFR pathway, including bevacizumab, ramucirumab, apatinib, and anlotinib. ${ }^{37}$ On the one hand, blockade of VEGF/VEGFR signaling pathway could lead to a decrease in nitric oxide production, causing vasculature constriction and a reduction in the renal excretion of sodium, which might contribute to an increase in blood pressure. ${ }^{38}$ On the other hand, hypertension could be a consequence of vascular rarefaction, which was the depletion of arterioles and capillaries resulting from the inhibition of the growth factors required to form new capillaries and recruit endothelial cell progenitors. ${ }^{39}$ Therefore, the occurrence of hypertension could partly reflect that anlotinib administration worked in the human body successfully and served as a biomarker eventually. However, the conclusion in our research should be interpreted with caution, given that elderly patients were always concomitant with potential predisposition factors that contributed to hypertension and most of them had a history of hypertension usually, which could compromise the true hypertension induced by anlotinib therapy. ${ }^{40}$ Consequently, our conclusion that elderly patients with hypertension might confer superior PFS should be validated in prospective clinical trials subsequently.

From the objective view, limitations existed in the present study inevitably. First, the sample size was small as a real-world study, and only 83 elderly patients were enrolled. Second, the present study was designed as a retrospective analysis and some bias could not be avoided. Clinical significance according to hypertension status was interpreted thoroughly. Collectively, we thought our study was of potential significance as the clinical guidance for elderly patients with treatment refractory NSCLC receiving anlotinib monotherapy.

\section{Disclosure}

The authors declare no conflicts of interest for this work.

\section{References}

1. Guo H, Wei J, Li X, et al. Do socioeconomic factors modify the effects of PM1 and SO2 on lung cancer incidence in China? Sci Total Environ. 2021;756:143998. doi:10.1016/j.scitotenv.2020.143998

2. Zhang J, Mao W, Chen Z, Gu H, Lian C. Clinical significance of Has_circ_0060937 in bone metastasis of NSCLC. Int J Gen Med. 2020;13:1115-1121. doi:10.2147/ijgm.s279023

3. Chen J, Chen J, Lv X, Yang Q, Yao S. Epidermal growth factor in exhaled breath condensate as diagnostic method for non-small cell lung cancer. Technol Cancer Res Treat. 2019;18:1533033819872271. doi:10.1177/1533033819872271

4. Jahanzeb M, Lin HM, Pan X, et al. Immunotherapy treatment patterns and outcomes among ALK-positive patients with non-small-cell lung cancer. Clin Lung Cancer. 2021;22(1):49-57. doi:10.1016/j. cllc. 2020.08 .003

5. Folkman J. Tumor angiogenesis: therapeutic implications. $N$ Engl $J \quad$ Med. 1971;285(21):1182-1186. doi:10.1056/nejm197111182 852108

6. Le X, Nilsson M, Goldman J, et al. Dual EGFR-VEGF pathway inhibition: a promising strategy for patients with EGFR-mutant NSCLC. $J$ Thorac Oncol. 2021;16(2):205-215. doi:10.1016/j. jtho.2020.10.006

7. Han B, Li K, Wang Q, et al. Effect of anlotinib as a third-line or further treatment on overall survival of patients with advanced non-small cell lung cancer: the ALTER 0303 Phase 3 Randomized Clinical Trial. JAMA Oncol. 2018;4(11):1569-1575. doi:10.1001/ jamaoncol.2018.3039

8. Blanco R, Maestu I, de la Torre MG, Cassinello A, Nuñez I. A review of the management of elderly patients with non-small-cell lung cancer. Ann Oncol. 2015;26(3):451-463. doi:10.1093/annonc/ mdu268 
9. Wang S, Liang Q, Chi Y, et al. Retrospective analysis of the effectiveness and tolerability of nab-paclitaxel in Chinese elderly patients with advanced non-small-cell lung carcinoma. Thorac Cancer. 2020;11(5):1149-1159. doi:10.1111/1759-7714.13356

10. Yamaguchi $\mathrm{O}$, Imai $\mathrm{H}$, Minemura $\mathrm{H}$, et al. Efficacy and safety of immune checkpoint inhibitor monotherapy in pretreated elderly patients with non-small cell lung cancer. Cancer Chemother Pharmacol. 2020;85(4):761-771. doi:10.1007/s00280-020-04055-7

11. Alexa T, Antoniu SA, Alexa I, et al. Checkpoint inhibitors in NSCLC for the elderly: current challenges and perspectives. Expert Rev Anticancer Ther. 2021;21(3):315-323. doi:10.1080/ 14737140.2021 .1852933

12. Zhong Y, Wei Q, Lu Y, et al. Efficacy and safety of anlotinib in patients with advanced non-small cell lung cancer. $J$ Thorac Dis. 2020;12(10):6016-6022. doi:10.21037/jtd-20-2855

13. Villaruz LC, Socinski MA. The role of anti-angiogenesis in non-small-cell lung cancer: an update. Curr Oncol Rep. 2015;17 (6):26. doi:10.1007/s11912-015-0448-y

14. Jayson GC, Kerbel R, Ellis LM, Harris AL. Antiangiogenic therapy in oncology: current status and future directions. Lancet. 2016;388 (10043):518-529. doi:10.1016/s0140-6736(15)01088-0

15. Song PF, Xu N, Li Q. Efficacy and safety of anlotinib for elderly patients with previously treated extensive-stage SCLC and the prognostic significance of common adverse reactions. Cancer Manag Res. 2020;12:11133-11143. doi:10.2147/cmar.s275624

16. Miller TP, Fisher BT, Getz KD, et al. Unintended consequences of evolution of the common terminology criteria for adverse events. Pediatr Blood Cancer. 2019;66:e27747. doi:10.1002/pbc.27747

17. Guo M, Li B, Yu Y, et al. Delineating the pattern of treatment for elderly locally advanced NSCLC and predicting outcomes by a validated model: a SEER based analysis. Cancer Med. 2019;8 (5):2587-2598. doi:10.1002/cam4.2127

18. Fukuda M, Soda H, Soejima Y, et al. A Phase I trial of carboplatin and etoposide for elderly ( $>$ or $=75$ year-old) patients with small-cell lung cancer. Cancer Chemother Pharmacol. 2006;58(5):601-606. doi:10.1007/s00280-006-0188-7

19. Yang Y, Sun N, Sun P, Zhang L. Clinical characteristics and prognosis of elderly small cell lung cancer patients complicated with hyponatremia: a retrospective analysis. Anticancer Res. 2017;37 (8):4681-4686. doi:10.21873/anticanres.11872

20. Bongiolatti S, Mazzoni F, Gonfiotti A, et al. Short and mid-term outcomes of multimodal treatment for locally-advanced non-small cell lung cancer in elderly patients. Gen Thorac Cardiovasc Surg. 2020;68(11):1290-1297. doi:10.1007/s11748-020-01384-5

21. Talarico L, Chen G, Pazdur R. Enrollment of elderly patients in clinical trials for cancer drug registration: a 7-year experience by the US Food and Drug Administration. J Clin Oncol. 2004;22 (22):4626-4631. doi:10.1200/jco.2004.02.175

22. Brozos-Vázquez EM, Díaz-Peña R, García-González J, et al. Immunotherapy in nonsmall-cell lung cancer: current status and future prospects for liquid biopsy. Cancer Immunol Immunother. 2021;70(5):1177-1188. doi:10.1007/s00262-020-02752-z

23. Schulz C, Gandara D, Berardo CG, et al. Comparative efficacy of second- and subsequent-line treatments for metastatic NSCLC: a fractional polynomials network meta-analysis of cancer immunotherapies. Clin Lung Cancer. 2019;20(6):451-460.e455. doi:10.1016/j.cllc.2019.06.017

24. Xie C, Wan X, Quan H, et al. Preclinical characterization of anlotinib, a highly potent and selective vascular endothelial growth factor receptor-2 inhibitor. Cancer Sci. 2018;109(4):1207-1219. doi:10.1111/cas.13536

25. Cheng Y, Wang Q, Li L, et al. OA13.03 Anlotinib as third-line or further-line treatment in relapsed SCLC: a multicentre, randomized, double-blind Phase 2 trial. J Thorac Oncol. 2018;13(10):S351-S352. doi:10.1016/j.jtho.2018.08.308
26. Chi Y, Fang Z, Hong X, et al. Safety and efficacy of anlotinib, a multikinase angiogenesis inhibitor, in patients with refractory metastatic soft-tissue sarcoma. Clin Cancer Res. 2018;24(21):5233-5238. doi:10.1158/1078-0432.ccr-17-3766

27. Li J, Chen P, Dai CH, Li XQ, Bao QL. Outcome and treatment in elderly patients with small cell lung cancer: a retrospective study. Geriatr Gerontol Int. 2009;9(2):172-182. doi:10.1111/j.14470594.2009.00525.x

28. Hu W, Li B, Geng N, et al. Association between PDL1 genetic variation and efficacy of apatinib monotherapy in patients with previously treated advanced NSCLC: a real-world retrospective study. Int J Gen Med. 2021;14:2703-2714. doi:10.2147/ijgm.s303717

29. Geng N, Su J, Liu Z, et al. The influence of KDR genetic variation on the efficacy and safety of patients with advanced NSCLC receiving first-line bevacizumab plus chemotherapy regimen. Technol Cancer Res Treat. 2021;20:15330338211019433. doi:10.1177/ 15330338211019433

30. Zhang K, Ma X, Gao H, et al. Efficacy and safety of anlotinib in advanced non-small cell lung cancer: a real-world study. Cancer Manag Res. 2020;12:3409-3417. doi:10.2147/cmar.s246000

31. Zhong Q, Liu Z. Efficacy and safety of anlotinib in patients with advanced non-small cell lung cancer: a real-world study. Cancer Manag Res. 2021;13:4115-4128. doi:10.2147/cmar.s304838

32. Cheng JD, Chai LX, Zhao ZP, Hao YY, Li S. Efficacy and safety of anlotinib for patients with advanced NSCLC who progressed after standard regimens and the preliminary analysis of an efficacy predictor. Cancer Manag Res. 2020;12:5641-5650. doi:10.2147/ cmar.s253366

33. Wang P, Fang X, Yin T, et al. Efficacy and safety of anti-PD-1 plus anlotinib in patients with advanced non-small-cell lung cancer after previous systemic treatment failure-A Retrospective Study. Front Oncol. 2021;11:628124. doi:10.3389/fonc.2021.628124

34. Wang J, Ma JJ, Liu J, et al. Prevalence and risk factors of comorbidities among hypertensive patients in China. Int J Med Sci. 2017;14 (3):201-212. doi:10.7150/ijms.16974

35. Fang SC, Huang W, Zhang YM, Zhang HT, Xie WP. Hypertension as a predictive biomarker in patients with advanced non-small-cell lung cancer treated with apatinib. Onco Targets Ther. 2019;12:985-992. doi:10.2147/ott.s189984

36. Liu X, Qin S, Wang Z, et al. Early presence of anti-angiogenesisrelated adverse events as a potential biomarker of antitumor efficacy in metastatic gastric cancer patients treated with apatinib: a cohort study. J Hematol Oncol. 2017;10(1):153. doi:10.1186/s13045-0170521-0

37. Bai ZG, Zhang ZT. A systematic review and meta-analysis on the effect of angiogenesis blockade for the treatment of gastric cancer. Onco Targets Ther. 2018;11:7077-7087. doi:10.2147/ott.s169484

38. Tang JR, Markham NE, Lin YJ, et al. Inhaled nitric oxide attenuates pulmonary hypertension and improves lung growth in infant rats after neonatal treatment with a VEGF receptor inhibitor. Am J Physiol Lung Cell Mol Physiol. 2004;287(2):L344-L351. doi:10.1152/ ajplung.00291.2003

39. Mourad JJ, des Guetz G, Debbabi H, Levy BI. Blood pressure rise following angiogenesis inhibition by bevacizumab. A crucial role for microcirculation. Ann Oncol. 2008;19(5):927-934. doi:10.1093/ annonc/mdm550

40. Oliveros E, Patel H, Kyung S, et al. Hypertension in older adults: assessment, management, and challenges. Clin Cardiol. 2020;43 (2):99-107. doi:10.1002/clc. 23303 


\section{Publish your work in this journal}

The International Journal of General Medicine is an international, peer-reviewed open-access journal that focuses on general and internal medicine, pathogenesis, epidemiology, diagnosis, monitoring and treatment protocols. The journal is characterized by the rapid reporting of reviews, original research and clinical studies across all disease areas. The manuscript management system is completely online and includes a very quick and fair peer-review system, which is all easy to use. Visit http://www.dovepress.com/ testimonials.php to read real quotes from published authors.

Submit your manuscript here: https://www.dovepress.com/international-journal-of-general-medicine-journal 